\title{
The central velocity dispersion of the Milky Way bulge ${ }^{\star}$
}

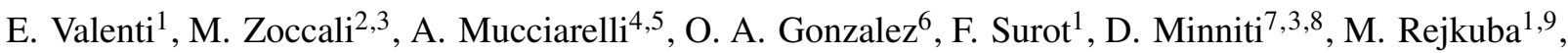 \\ L. Pasquini ${ }^{1}$, G. Fiorentino ${ }^{5}$, G. Bono ${ }^{10,11}$, R. M. Rich ${ }^{12}$, and M. Soto ${ }^{13,14}$ \\ ${ }^{1}$ European Southern Observatory, Karl Schwarzschild-Straße 2, 85748 Garching bei München, Germany \\ e-mail: evalenti@eso.org \\ 2 Instituto de Astrofísica, Pontificisa Universidad Católica de Chile, Av. Vicuña Mackenna 4860, Santiago, Chile \\ 3 Millennium Institute of Astrophysics, Av. Vicuña Mackenna 4860, 782-0436 Macul, Santiago, Chile \\ ${ }^{4}$ Dipartimento di Fisica e Astronomia - Universitá degli Studi di Bologna, Via Piero Gobetti 93/2, 40129 Bologna, Italy \\ 5 INAF - Osservatorio di Astrofisica e Scienza dello Spazio di Bologna, Via Piero Gobetti 93/3, 40129 Bologna, Italy \\ ${ }^{6}$ UK Astronomy Technology Centre, Royal Observatory, Blacford Hill, Edinburgh EH9 3HJ, UK \\ Departamento de Ciencias Fisícas, Universidad Andrés Bello, República 220, Santiago, Chile \\ Vatican Observatory, V00120 Vatican City State, Italy \\ 9 Excellence Cluster Universe, Boltzmann-Straße 2, 85748 Garching bei München, Germany \\ 10 Department of Physics, University of Roma Tor Vergata, Via della Ricerca Scientifica 1, 00133 Roma, Italy \\ 11 INAF - Osservatorio Astronomico di Roma, Via Frascati 33, 00040 Monte Porzio Catone RM, Italy \\ 12 Division of Astronomy, Department of Physics and Astronomy, UCLA, PAB 430 Portola Plaza, Los Angeles, \\ CA 90095-1547, USA \\ 13 Universidad de Atacama, Departamento de Física, Copayapu 485, Copiapó, Chile \\ 14 Space Telescope Science Institute, San Martin Drive 3700, Baltimore, MD 21218, USA
}

Received 26 February 2018 / Accepted 30 April 2018

\begin{abstract}
Context. Current spectroscopic and photometric surveys are providing a comprehensive view of the Milky Way bulge stellar population properties with unprecedented accuracy. This in turn allows us to explore the correlation between kinematics and stellar density distribution, crucial to constrain the models of Galactic bulge formation.

Aims. The Giraffe Inner Bulge Survey (GIBS) revealed the presence of a velocity dispersion peak in the central few degrees of the Galaxy by consistently measuring high velocity dispersion in the three central most fields. Due to the suboptimal distribution of these fields, all being at negative latitudes and close to each other, the shape and extension of the sigma peak is poorly constrained. In this study we address this by adding new observations distributed more uniformly and in particular including fields at positive latitudes that were missing in GIBS.

Methods. Observations with Multi Unit Spectroscopic Explorer (MUSE) were collected in four fields at $(l, b)=\left(0^{\circ},+2^{\circ}\right),\left(0^{\circ},-2^{\circ}\right)$, $\left(+1^{\circ},-1^{\circ}\right)$, and $\left(-1^{\circ},+2^{\circ}\right)$. Individual stellar spectra were extracted for a number of stars comprised between $\sim 500$ and $\sim 1200$, depending on the seeing and the exposure time. Velocity measurements are done by cross-correlating observed stellar spectra in the $\mathrm{CaT}$ region with a synthetic template, and velocity errors are obtained through Monte Carlo simulations, cross-correlating synthetic spectra with a range of different metallicities and different noise characteristics.

Results. We measure the central velocity dispersion peak within a projected distance from the Galactic center of $\sim 280 \mathrm{pc}$, reaching $\sigma V_{\mathrm{GC}} \sim 140 \mathrm{~km} \mathrm{~s}^{-1}$ at $b=-1^{\circ}$. This is in agreement with the results obtained previously by GIBS at negative longitude. The central sigma peak is symmetric with respect to the Galactic plane, with a longitude extension at least as narrow as predicted by GIBS. As a result of the Monte Carlo simulations we present analytical equations for the radial velocity measurement error as a function of metallicity and signal-to-noise ratio for giant and dwarf stars.
\end{abstract}

Key words. Galaxy: bulge - techniques: imaging spectroscopy

\section{Introduction}

Current photometric and spectroscopic surveys of the Galactic bulge are providing a wealth of data to explore the spatial distribution, chemical content, and kinematics of its stellar population. A special 2016 edition of the Publications of the Astronomical Society of Australia (vol. 33) provides several reviews on the bulge observational properties (e.g., Zoccali \& Valenti 2016; Babusiaux 2016).

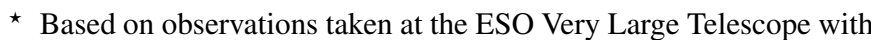
the MUSE instrument under program IDs 060.A-9342 (Science Verification; PI: Valenti/Zoccali/Kuijken), and 99.B-0311A (SM; PI: Valenti).
}

Stellar kinematics and spatial distribution in particular are thought to be strongly correlated with the bulge formation process. Two main scenarios have been proposed for bulge formation. The first one is through evolution of the disk, when the latter has been mostly converted into stars. In this case the bulge is expected to have the shape of a bar, though vertically heated into a boxy/peanut shape, with corresponding kinematics. The second scenario is the hierarchical merging of gas rich sub-clumps coming either from the disk or from satellite structures. In this case both the spatial distribution and the kinematics are expected to be more isotropic. Obviously, a combination of these two scenarios could have also led to the formation of the Galactic bulge we observe today. 


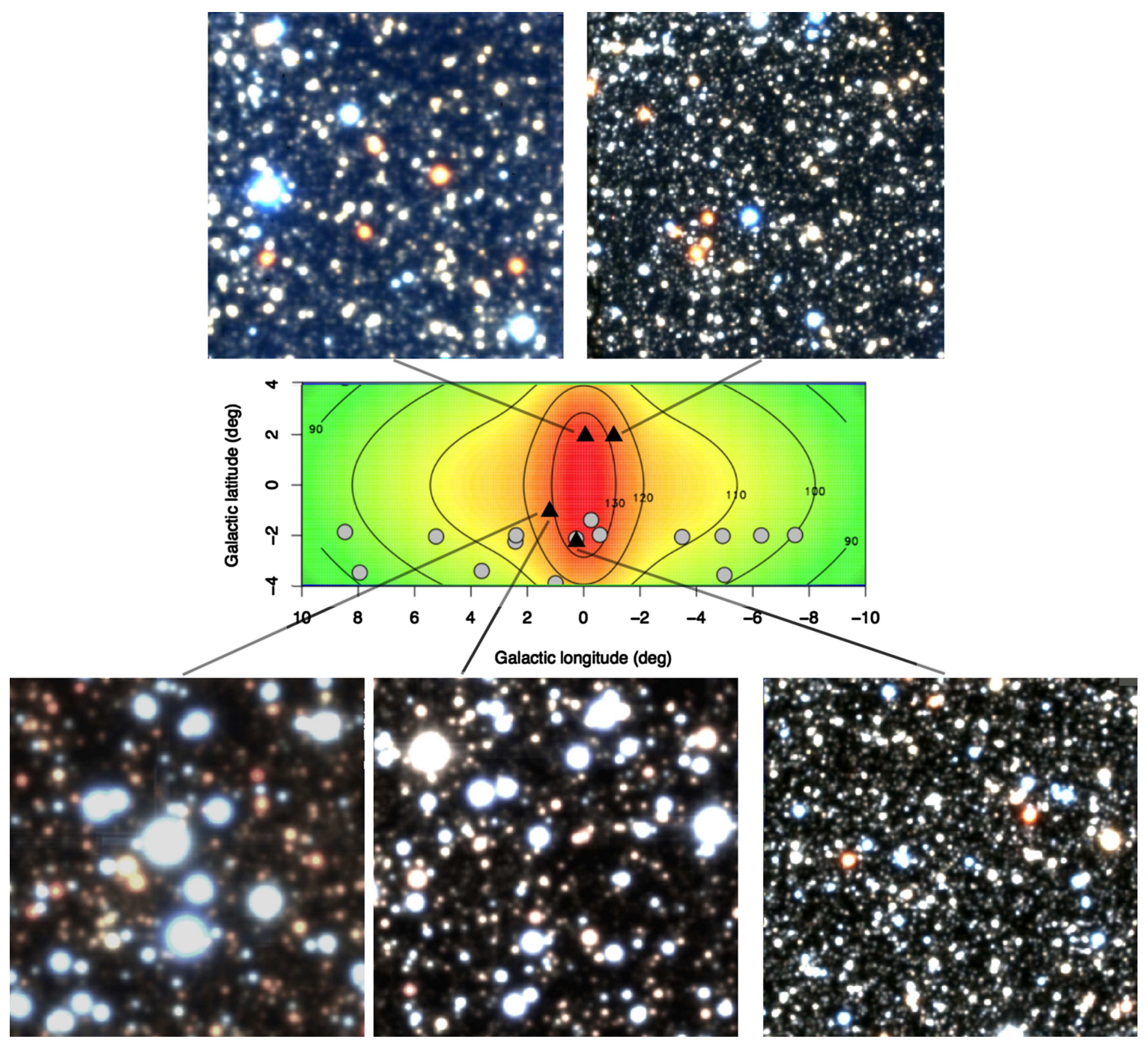

Fig. 1. VRI MUSE FOV colored images of the bulge $1^{\prime} \times 1^{\prime}$ fields analyzed here, from top left clockwise: p0p2, m1p2, p0m2, and two pointings at $1 \mathrm{~m} 1$ highlighting the impact of different image quality. Their Galactic position relative to the velocity dispersion map of Zoccali et al. (2014) is also shown.

Early kinematical surveys covering a large bulge area such as the Bulge RAdial Velocity Assay (Rich et al. 2007; Howard et al. 2009) and the Abundances and Radial velocity Galactic Origin Survey (Freeman et al. 2013; Ness et al. 2013a,b) derived a rotation curve that looked cylindrical, supporting the conclusion that the bulge had been formed exclusively via disk dynamical instabilities (Shen et al. 2010). These studies, however, were limited - by crowding and interstellar extinction - to latitudes $|b|>4^{\circ}$. By using data from the Giraffe Inner Bulge Survey (GIBS), Zoccali et al. (2014) found that the radial velocity dispersion $(\sigma)$ exhibits a strong increase resulting in a peak with $\sim 140 \mathrm{~km} \mathrm{~s}^{-1}$, confined within a radius of $\sim 250$ pc from the Galactic center. It was later demonstrated that this peak is spatially associated to a peak in star counts (Valenti et al. 2016), hence in stellar mass, and it is slightly dominated by metal-poor stars (Zoccali et al. 2017).

Indeed, there is now consensus that the inner Galactic bulge hosts two components that are best separated in metallicity $([\mathrm{Fe} / \mathrm{H}])$, but also show different spatial distribution (Ness et al. 2012; Dékány et al. 2013; Rojas-Arriagada et al. 2014; Pietrukowicz et al. 2015; Gran et al. 2016; Zoccali et al. 2017), kinematics (Babusiaux et al. 2010; Zoccali et al. 2017), and $[\mathrm{Mg} / \mathrm{Fe}]$ ratio (Hill et al. 2011).
The velocity dispersion peak found from GIBS data was constrained by three fields, at Galactic coordinates $(l, b)=\left(-0.26^{\circ}\right.$, $\left.-1.40^{\circ}\right),\left(0.27^{\circ},-2.13^{\circ}\right)$, and $\left(-0.58^{\circ},-1.98^{\circ}\right)$, respectively. The velocity dispersion was derived from samples of 441,435 , and 111 stars, respectively. The need to obtain intermediate resolution optical spectra for many stars, with a signal-to-noise ratio $(\mathrm{S} / \mathrm{N})$ high enough to allow us to measure Calcium II Triplet (CaT) metallicity, restricted the position of the GIBS innermost fields to the bulge hemisphere at negative latitudes. In order to constrain the shape and spatial extension of the $\sigma$-peak, we analyze here new data obtained with the MUSE integral field spectrograph (IFS) at the ESO Very Large Telescope (VLT), in fields closer to the Galactic center at both positive and negative latitudes.

\section{Observations and data reduction}

Three fields, hereafter named $\mathrm{p} 0 \mathrm{~m} 2, \mathrm{p} 0 \mathrm{p} 2$, and $\mathrm{m} 1 \mathrm{p} 2$, located in the innermost bulge regions were observed with MUSE during the Science Verification campaign. Another one, consisting of two adjacent pointings, named p1m1-A and p1m1-B, was observed in service mode as part of a filler program 99.B-0311A (PI: Valenti) for which only $4 \mathrm{~h}$ were executed of the $76 \mathrm{~h}$ orig- 
Table 1. Galactic coordinates, reddening, image quality, and observations log of the observed fields.

\begin{tabular}{lccccc}
\hline \hline Field & $l$ & $b$ & Exp. time & $F W H M$ & $\mathrm{E}(J-K s)$ \\
\hline p0m2 & $+0.26^{\circ}$ & $-2.14^{\circ}$ & $6 \times 1000 \mathrm{~s}$ & $0.6^{\prime \prime}$ & 0.36 \\
m1p2 & $-1.00^{\circ}$ & $+2.00^{\circ}$ & $2 \times 1000 \mathrm{~s}$ & $0.5^{\prime \prime}$ & 0.86 \\
p0p2 & $0.00^{\circ}$ & $+2.00^{\circ}$ & $3 \times 1000 \mathrm{~s}$ & $1.1^{\prime \prime}$ & 0.90 \\
p1m1-A & $+1.20^{\circ}$ & $-1.00^{\circ}$ & $6 \times 1066 \mathrm{~s}$ & $1.2^{\prime \prime}$ & 0.87 \\
p1m1-B & $+1.20^{\circ}$ & $-1.00^{\circ}$ & $6 \times 1066 \mathrm{~s}$ & $0.9^{\prime \prime}$ & 0.86 \\
\hline
\end{tabular}

inally approved. Table 1 lists the Galactic coordinates, exposure times, image quality, and interstellar extinction (Gonzalez et al. 2012) for all the fields. The two pointings of the $\mathrm{p} 1 \mathrm{~m} 1$ field were observed under quite different seeing conditions, but they are so close to each other that they have the same velocity dispersion, and are thus treated as a single field hereafter.

MUSE (Bacon et al. 2010) is the integral field spectrograph at the Nasmyth B focus of the Yepun (VLT-UT4) telescope at the ESO Paranal Observatory. It provides 1 square arcmin field of view, with a spatial pixel of $0.2^{\prime \prime}$, and a mean spectral resolution of $R \approx 3000$. The observations were carried out in seeing limited mode (WFM-noAO) by using the so-called Nominal setup, which yields a continuous wavelength coverage between $4750 \AA$ and $9350 \AA$.

For all the fields, we used similar observing strategy but different total integration time (see Table 1): a combination of on-target sub-exposures each $\sim 1000 \mathrm{~s}$ long, taken with a small offsets pattern (i.e., $\sim 1.5^{\prime \prime}$ ) and $90^{\circ}$ rotations in order to optimize the cosmics rejection and obtaining a uniform combined dataset in terms of noise properties.

The processing of the raw data was performed with the MUSE pipeline (v.1.5, Weilbacher et al. 2012). The entire pipeline data reduction cascade consists of two main steps: (i) creating all necessary calibrations to remove the instrument signature from each target exposures, such as bias, flats, bad pixels map, instrument geometry, illumination, astrometry correction, line spread function, response curve for flux calibration, and wavelength solution map; and (ii) constructing, for each target field, the final datacube by combining the different science exposures processed during the previous step. In addition to the final datacube, the pipeline optionally produces the so-called field-ofview (FoV) images by convolving the MUSE datacube with the transmission curve of various filters. For this work we produced FoV images in $V$-Johnson, $R$-Cousins, and $I$-Cousins. Figure 1 shows the color image of each target field obtained combining the $V, R$, and $I$ FoV images, and the position of the four fields in the velocity dispersion map provided by the GIBS survey. Clearly, the number of stars detected in each field is affected both by the different seeing conditions and by the extinction of the field.

Extraction of the spectra. The procedure adopted to extract the spectra for all the stellar sources present in the target fields consists of two main steps: (i) the creation of a master star list for each field; and (ii) the reconstruction of the spectrum of each star in the list, by using the star flux as measured in the MUSE final data cubes.

We first performed standard aperture photometry, with DAOPHOT (Stetson 1987), on the FoV images to obtain a master list of all sources with significant counts $(>4 \sigma)$ above the background. Due to the relatively modest crowding, aperture or point spread function (PSF)-fitting photometry yield virtually
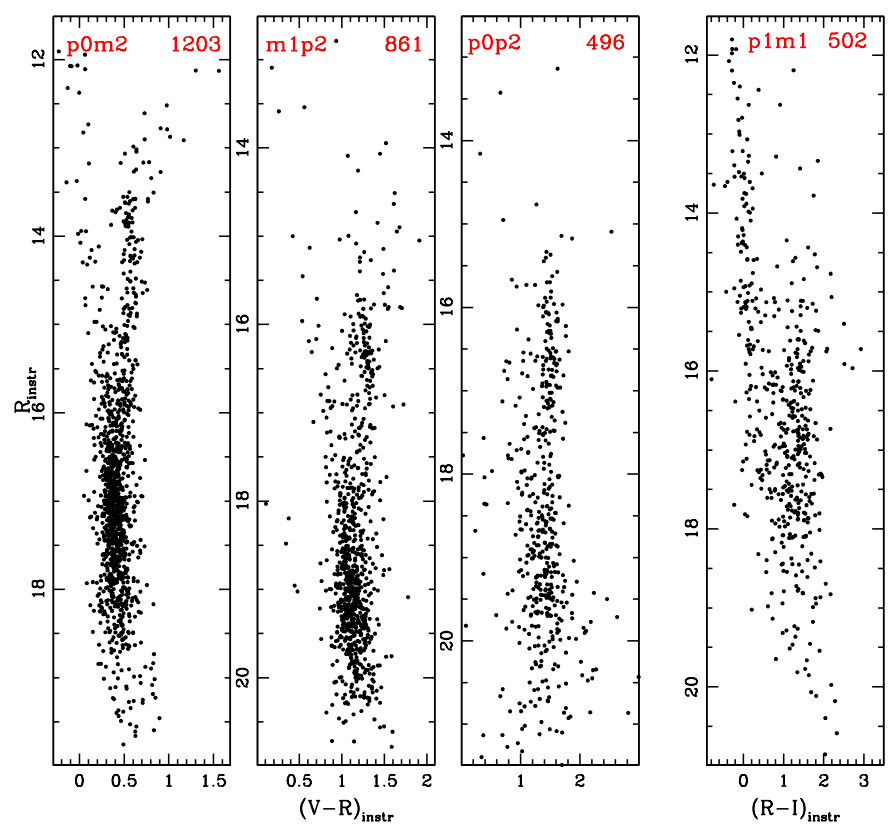

Fig. 2. Instrumental CMD of the observed bulge fields as derived by running aperture photometry on the MUSE FoV images. The name of the fields and total number of detected stars are given.

identical results, therefore we used aperture photometry hereafter (see below). Figure 2 shows the derived color-magnitude diagrams (CMDs) for all the fields, either in the $(R, V-R)$ or in the $(R, R-I)$ instrumental plane. The latter was used for the p1m1 field because, due to its higher extinction, the $V$ image had the lowest $\mathrm{S} / \mathrm{N}$. Here the impact of the different seeing and total exposure time is also very clear, with the p0p2 field being the least populated due to the combination of relatively poor seeing and short total exposure time. The $\mathrm{p} 1 \mathrm{~m} 1$ field is the closest one to the Galactic plane, therefore showing a prominent disk main sequence (MS) that is both more prominent and extends to brighter magnitudes in comparison to the other fields. This is due to the fact that at $b=-1^{\circ}$ the optical depth of the thin disk is larger. The presence of bright blue stars is very evident also in the FoV images of Fig. 1.

The final MUSE data cubes were then sliced along the wavelength axis into 3681 monochromatic images (i.e., single planes) sampling the target stars from $4750 \AA$ to $9350 \AA$ with a step in wavelength of $1.25 \AA$. Aperture photometry was performed on each of these images (task PHOT of IRAF $^{1}$ ) with an aperture radius $\sim 1.5 \times\langle F W H M\rangle$, where $\langle\mathrm{FWHM}\rangle$ is the average image quality measured over the wavelength range $\lambda>6000 \AA$. Finally, for each star in the master list, the corresponding spectrum was obtained by assigning to each wavelength the flux measured on the corresponding monochromatic image. For a given field, a single value was used for the aperture radius, since the full width half maximum (FWHM) variation, as measured across the entire wavelength range, is about half a pixel, independent from the mean FWHM value.

It is worth mentioning that this photometric approach to the extraction of IFS spectra has the advantage of successfully

1 IRAF is distributed by the National Optical Astronomy Observatories, which are operated by the Association of Universities for Research in Astronomy, Inc., under cooperative agreement with the National Science Foundation. 


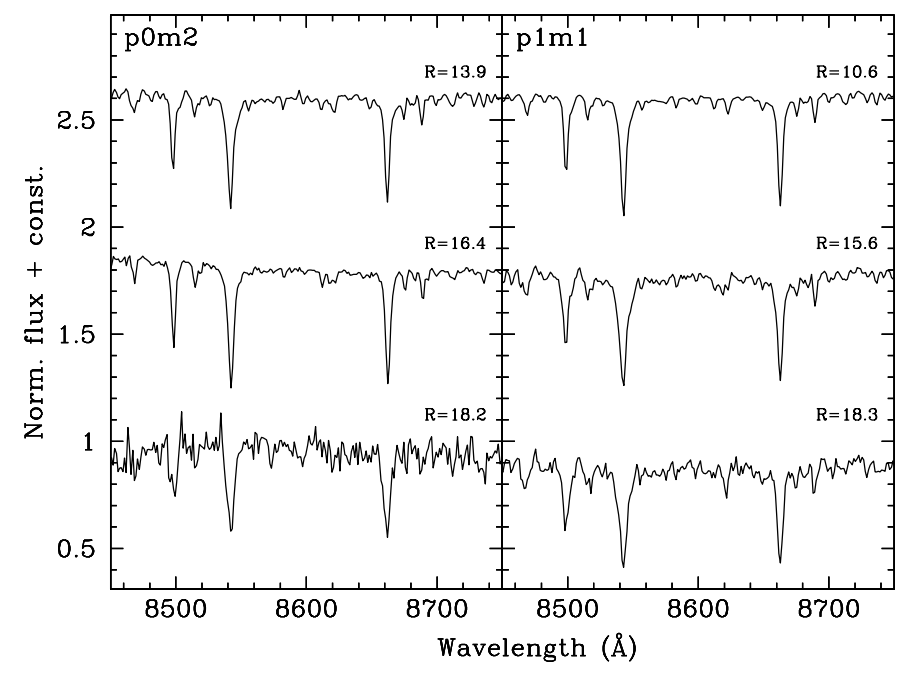

Fig. 3. Example of typical spectra in the CaT region, for stars in the brightest $0.5 \mathrm{mag}$ bin, for red clump stars, and stars at the very faintend magnitude range. For each spectrum, the instrumental $R$ magnitude of the star is given.

addressing the issue of sky subtraction residuals often present in the final datacubes. Indeed, it is well known that the sky subtraction may not always be optimal, leading to the presence of artifacts (e.g., weak emission line residuals and/or $p$ cygni-like profiles) in the final spectra. By contrast, any such residuals present in the single plane images are fully taken into account by the photometric procedure, which estimates a local sky background for each source present in the master list.

Several attempts at using PSF-fitting photometry on the monochromatic images were performed. They were finally discarded because the majority of the stars were lost in a few of the monochromatic images, corresponding to the bottom of their strongest absorption lines. When the star flux is close to the sky level, aperture photometry still assigns a meaningful flux value, while PSF-fitting photometry just discards the star from the list. This is not a negligible issue, given that the strong absorption lines are very important in the measurement of radial velocities. On the other hand, given the modest crowding of the images, the photometry from aperture and PSF-fitting yielded similar quality results, at least on the FoV images. Therefore we judged it unnecessary, in this case, to try and overcome the problem of non-convergence of the PSF in the low signal regime. The analysis of more crowded fields such as the inner regions of dense star clusters might require a different approach.

Spectra for $1203,861,496$, and 502 stars were reconstructed in the $\mathrm{p} 0 \mathrm{~m} 2, \mathrm{~m} 1 \mathrm{p} 2, \mathrm{p} 0 \mathrm{p} 2$, and $\mathrm{p} 1 \mathrm{~m} 1$ fields, respectively. Examples of typical extracted spectra, zoomed in the CaT region, for stars of different magnitudes are given in Fig. 3. We show spectra for the field with the best combination of seeing and exposure time (p0m2) and the one with the worst seeing (p1m1-A). The average $\mathrm{S} / \mathrm{N}$, as measured in the CaT wavelength range, of field $\mathrm{p} 0 \mathrm{~m} 2, \mathrm{~m} 1 \mathrm{p} 2, \mathrm{p} 0 \mathrm{p} 2$, and $\mathrm{p} 1 \mathrm{~m} 1$ stars in the faintest 0.5 mag bin, is $\sim 20,15,10,10$ respectively.

\section{Radial velocities and velocity errors}

We measured the heliocentric radial velocity (RV) of all stars detected in the observed fields through cross-correlation with a synthetic template by using the IRAF task fxcor. Specifically, we adopted for all stars the same synthetic spectra of a relatively

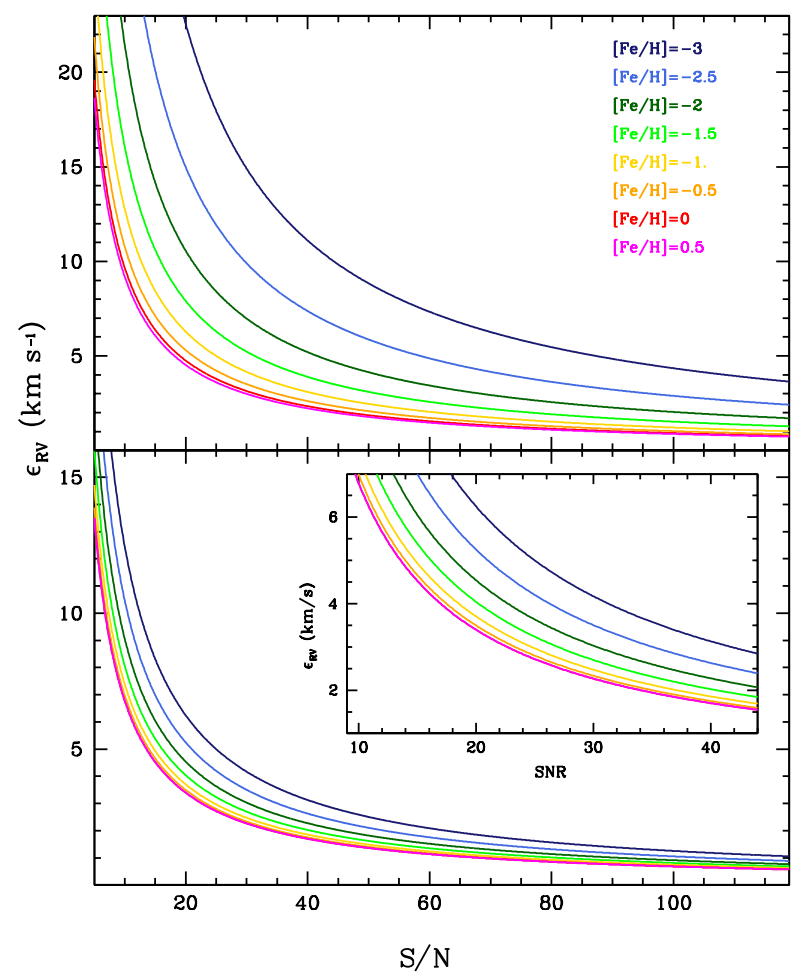

Fig. 4. RV error $\left(\epsilon_{\mathrm{RV}}\right)$ profile as a function of the spectra $\mathrm{S} / \mathrm{N}$ for giants (bottom panel) and dwarfs (top panel) of different metallicity. The inset shows the $\epsilon_{\mathrm{RV}}$ profile of giants in the medium-low $\mathrm{S} / \mathrm{N}$ regime.

metal rich $([\mathrm{Fe} / \mathrm{H}]=-0.4$ dex $) \mathrm{K}$ giant and performed the crosscorrelation between the model and the observed spectra in the wavelength range bracketing the CaT lines. To assess the effect that the use of a single metallicity template may have on the derived velocities, in the case of p0m2 stars field we also used two additional synthetic templates with metallicities $([\mathrm{Fe} / \mathrm{H}]=$ -1.3 dex and +0.2 dex) that bracket the typical metallicity distribution function observed in the GIBS fields by Zoccali et al. (2017). We found that the RV derived with the metal-poor and metal-rich models always agree within $\leq 1 \mathrm{~km} \mathrm{~s}^{-1}$, thus confirming what had already been noticed by Zoccali et al. (2014) that the metallicity of the adopted synthetic template has a very minor effect on the derived RV.

The error in the derived RV could not be estimated from repeated measurements because, for each target field, we only have one single datacube. Therefore, the uncertainty $\left(\epsilon_{\mathrm{RV}}\right)$ has been estimated by means of Monte Carlo simulations. The main sources of uncertainties are the $\mathrm{S} / \mathrm{N}$, the spectral resolution, and the sampling. In order to evaluate their impact on the derived RVs we generated different sets of artificial MUSE spectra, varying $\mathrm{S} / \mathrm{N}$ and metallicity, reproducing the observed ones. We started from synthetic spectra calculated with the code SYNTHE (Sbordone et al. 2004), assuming typical parameters of a giant $\left(T_{\text {eff }}=4500 \mathrm{~K}, \log g=1.5, v_{\text {turb }}=2 \mathrm{~km} \mathrm{~s}^{-1}\right)$ and a dwarf star $\left(T_{\text {eff }}=6500 \mathrm{~K}, \log g=4.5, v_{\text {turb }}=1 \mathrm{~km} \mathrm{~s}^{-1}\right)$, and considering a grid of metallicity between $[\mathrm{Fe} / \mathrm{H}]=-3.0$ and +0.5 dex with a step of $0.5 \mathrm{dex}$. These synthetic spectra have been convolved with a Gaussian profile to reproduce the spectral resolution of MUSE and then resampled at the same pixel size of the observed extracted spectra $\left(1.25 \AA\right.$ pixel $\left.^{-1}\right)$. Poisson noise was added to the synthetic spectra in order to reproduce different noise conditions, from $S / N \sim 10$ to $S / N \sim 100$ with steps of 10 . At the end, for each metallicity and $\mathrm{S} / \mathrm{N}$, a sample of 500 synthetic 
spectra with randomly added noise was generated and their RVs measured through a cross-correlation technique (fxcor) with the original synthetic spectrum as template.

The dispersion of the derived RVs of each sample has been assumed as $1 \sigma$ uncertainty in the RV measurement for a given $\mathrm{S} / \mathrm{N}$ and metallicity. We derived the following relations that link the radial velocity error to $\mathrm{S} / \mathrm{N}$ and metallicity for giant stars:

$\ln \left(\epsilon_{\mathrm{RV}}\right)=4.209-0.997 \ln (\mathrm{S} / \mathrm{N})-0.029[\mathrm{Fe} / \mathrm{H}]+0.058[\mathrm{Fe} / \mathrm{H}]^{2}$,

and for dwarf stars:

$\ln \left(\epsilon_{\mathrm{RV}}\right)=4.624-1.023 \ln (\mathrm{S} / \mathrm{N})-0.159[\mathrm{Fe} / \mathrm{H}]+0.120[\mathrm{Fe} / \mathrm{H}]^{2}$.

The behavior of $\epsilon_{\mathrm{RV}}$ as a function of $\mathrm{S} / \mathrm{N}$ for different values of $[\mathrm{Fe} / \mathrm{H}]$ is shown in Fig. 4 for giant and dwarf stars. For giant stars $\epsilon_{\mathrm{RV}}$ increases rapidly for $\mathrm{S} / \mathrm{N}$ smaller than 30, reaching uncertainties at $S / N=10$ of 6.8 and $12.5 \mathrm{~km} \mathrm{~s}^{-1}$ for $[\mathrm{Fe} / \mathrm{H}]=+0.5$ and -3.0 dex, respectively, while at high $\mathrm{S} / \mathrm{N}, \epsilon_{\mathrm{RV}}$ is almost constant and close to $\sim 1 \mathrm{~km} \mathrm{~s}^{-1}$. At a given $\mathrm{S} / \mathrm{N}, \epsilon_{\mathrm{RV}}$ increases as $[\mathrm{Fe} / \mathrm{H}]$ decreases, due to the weakening of the CaT lines, while for $[\mathrm{Fe} / \mathrm{H}]$ larger than -1.0 dex the curves are almost indistinguishable. A similar general behavior is also found for the dwarf stars, but with larger uncertainties because of the weakness of the CaT lines: in particular at $S / N=10$, the relation provides $\epsilon_{\mathrm{RV}}=9.2 \mathrm{~km} \mathrm{~s}^{-1}$ for $[\mathrm{Fe} / \mathrm{H}]=+0.5 \mathrm{dex}$, while at lower metallicities the uncertainties increase dramatically (up to $\sim 45 \mathrm{~km} \mathrm{~s}^{-1}$ for $[\mathrm{Fe} / \mathrm{H}]=-3.0 \mathrm{dex}$ ). We limited this procedure to the $\mathrm{CaT}$ lines spectral region, in a window between $8450 \AA$ and $8700 \AA$, therefore these relations are specific to the case of MUSE in this spectral window.

For each field, Table 2 lists the errors on the RV estimates for stars in the faintest $0.5 \mathrm{mag}$ bin as derived by using the above mentioned relations for two different metallicity values: $[\mathrm{Fe} / \mathrm{H}]=$ $-1 \mathrm{dex}$ and $[\mathrm{Fe} / \mathrm{H}]=+0.5 \mathrm{dex}$, which represent the metal-poor and metal-rich edge of the typical bulge metallicity distribution function. In particular, due to the differences in the magnitude depth among the different fields, the values quoted in Table 2 have been derived by using Eq. (2) for the p0m2, m1p2, and p0p2 fields, whereas for $\mathrm{p} 1 \mathrm{~m} 1$ we have adopted the relation for giants (i.e., Eq. (1)). As expected, we found that at fixed $\mathrm{S} / \mathrm{N}$ (i.e., magnitude) metal-rich stars have typically smaller radial velocity error. However, the variation over the entire bulge metallicity range is $\leq 2 \mathrm{~km} \mathrm{~s}^{-1}$ (see Table 2 and Fig. 4).

\section{Velocity dispersion}

In order to measure the velocity dispersion of bulge stars, it is important to take into account the contamination by foreground disk stars, which are known to have a smaller velocity dispersion (Ness et al. 2016; Robin et al. 2017). An estimate of the actual disk velocity dispersion can be attempted by selecting foreground disk MS stars in the instrumental CMD of each observed field. This is shown in Figs. 5-8. Thanks to the good seeing, longer exposure time, and relatively low reddening, the p0m2 field has the best defined CMD, reaching fainter magnitudes (Fig. 5). In this field, bona-fide bulge-red giant branch (RGB)/disk-MS stars (red/blue symbols, respectively) are selected as having both $R<16$ and $(V-R)$ larger/smaller than 0.35 , respectively. The cuts isolate 75 disk MS stars, shown in blue in the CMD, and 206 bulge RGB stars shown in red. Stars
Table 2. Typical radial velocity error of observed stars in the faintest $0.5 \mathrm{mag}$ bin as derived from Eq. 2 for $[\mathrm{Fe} / \mathrm{H}]=$ $-1 \mathrm{dex}(\mathrm{MP})$ and $[\mathrm{Fe} / \mathrm{H}]=+0.5 \mathrm{dex}(\mathrm{MR})$.

\begin{tabular}{lccc}
\hline \hline Field & $S / N$ & $\begin{array}{c}\epsilon_{\mathrm{RV}}(\mathrm{MP}) \\
\mathrm{km} \mathrm{s}^{-1}\end{array}$ & $\begin{array}{c}\epsilon_{\mathrm{RV}}(\mathrm{MR}) \\
\mathrm{km} \mathrm{s}^{-1}\end{array}$ \\
\hline p0m2 & 20 & 6.3 & 4.5 \\
m1p2 & 15 & 8.4 & 6.1 \\
p0p2 & 10 & 12.8 & 9.2 \\
p1m1 $^{a}$ & 10 & 7.4 & 6.8 \\
\hline
\end{tabular}

Notes. ${ }^{(a)}$ For this field the faintest stars are giants, therefore we have used Eq. (1).

fainter than $R=16$, plotted in green, cannot be safely assigned to either population. The top-right panels of Figs. 5-8 shows the heliocentric radial velocity versus magnitude for all the stars, with the same color-coding as before. It is clear that disk MS stars have a lower velocity dispersion but, as expected, their radial velocity distribution is contaminated by bulge stars, both blue stragglers and subgiant branch stars. In fact, the radial velocity histogram shown at the bottom of the right panel clearly shows the presence of outliers at $|R V|>150 \mathrm{~km} \mathrm{~s}^{-1}$. Indeed, if these stars are excluded, by a simple cut at $|R V|<120 \mathrm{~km} \mathrm{~s}^{-1}$ the radial velocity dispersion drops to a value of $\sigma_{\mathrm{RV}}=45 \mathrm{~km} \mathrm{~s}^{-1}$, consistent in all three fields at $b= \pm 2^{\circ}$.

This exercise allows us to conclude that in the region of the CMDs above the old MS turnoff, where we can safely separate disk foreground from bulge stars by means of a color cut, the velocity dispersion of the disk is significantly lower than that of the bulge. Therefore, in order to include bulge MS stars in our analysis, we need to allow for the presence of two components with different kinematics.

In the field at $b=-1^{\circ}$ the data do not reach the bulge MS, and therefore the foreground disk MS and the bulge RGB can be separated by just a color cut at $(R-I)_{\text {inst }}=0.7$. In Fig. 8 , the panels on the right show that disk stars, with the same selection imposed for the other fields $\left(|R V|<120 \mathrm{~km} \mathrm{~s}^{-1}\right)$, have a velocity dispersion of $39 \mathrm{~km} \mathrm{~s}^{-1}$. This value is lower than the $45 \mathrm{~km} \mathrm{~s}^{-1}$ found at $b= \pm 2^{\circ}$, consistent with the fact that this field, at $b=-1^{\circ}$, samples more thin disk stars, having a smaller velocity dispersion. Bulge RGB stars, on the other hand, have a velocity dispersion $\sigma=119 \mathrm{~km} \mathrm{~s}^{-1}$. This value will not be further refined, because it already includes all the bulge stars measured in this field, with a negligible contamination from disk stars.

The RV distributions for all the sampled stars, in the three fields at $b= \pm 2^{\circ}$, are shown in Fig. 9. As demonstrated above, the best fit to the observed velocity distribution in all fields is obtained with a combination of two Gaussian components with approximately the same mean $\left(\left(R V_{\mathrm{b}}-R V_{\mathrm{d}}\right) \sim 10 \mathrm{~km} \mathrm{~s}^{-1}\right)$ but very different $\sigma$. The foreground disk stars contaminating the bulge sample have a velocity distribution with smaller dispersion (blue dashed line in Fig. 9). Specifically, we found the velocity dispersion of the disk population along the line of sight towards $\mathrm{p} 0 \mathrm{~m} 2, \mathrm{~m} 1 \mathrm{p} 2$, and $\mathrm{p} 0 \mathrm{p} 2$ fields to be $\sigma=45,40$, and $35 \mathrm{~km} \mathrm{~s}^{-1}$, respectively. These values agree with the Apache Point Observatory Galactic Evolution Experiment findings (Ness et al. 2016) for disk stars in the foreground of the bulge, within a distance of $3 \mathrm{kpc}$, as well as those reported by Robin et al. (2017) for thick disk stars in the solar neighborhood. In Table 3 we list the mean heliocentric RV and velocity dispersion measured for the bulge stars as obtained from the best fit to the velocity 

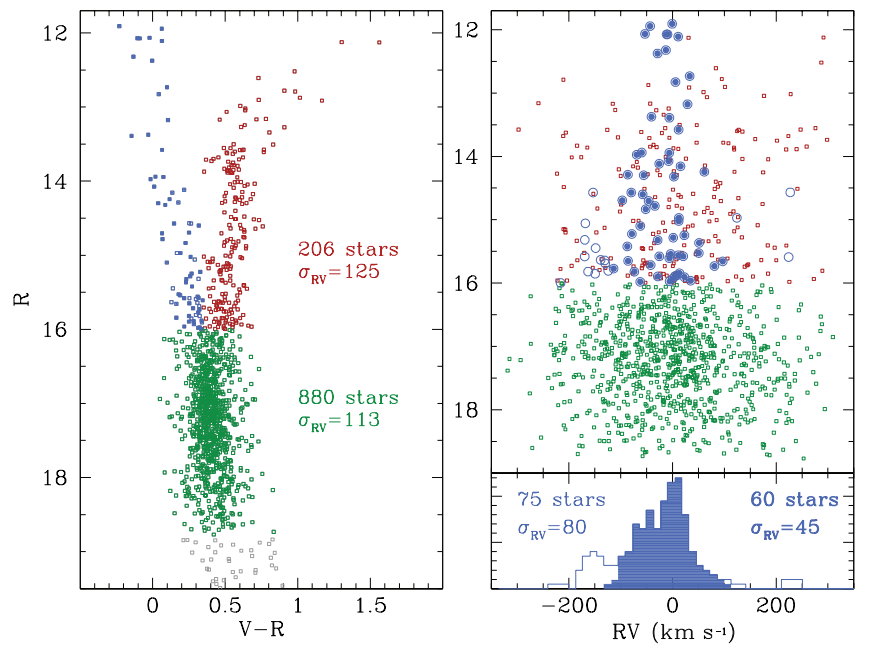

Fig. 5. Left panel: CMD of the global targets sampled in p0m2 colorcoded according to their evolutionary phase. Red and blue symbols refer to the bona-fide bulge-RGB and disk-MS stars, respectively. For the bona-fide sample, the total number of stars and their radial velocity dispersion are also given. Green circles mark either bulge- or disk-MS and MS-TO stars. Top right panel: heliocentric RV as a function of the star magnitude of all stars adopting the same color code as in the left panel. Blue solid symbols refer to disk-MS stars with $|R V|<120 \mathrm{~km} \mathrm{~s}^{-1}$. Bottom right panel: heliocentric radial velocity distribution of disk-MS stars. The velocity dispersion of the total disk-MS sample and of the sub-sample obtained after applying a cut at $|R V|<120 \mathrm{~km} \mathrm{~s}^{-1}$ are given.
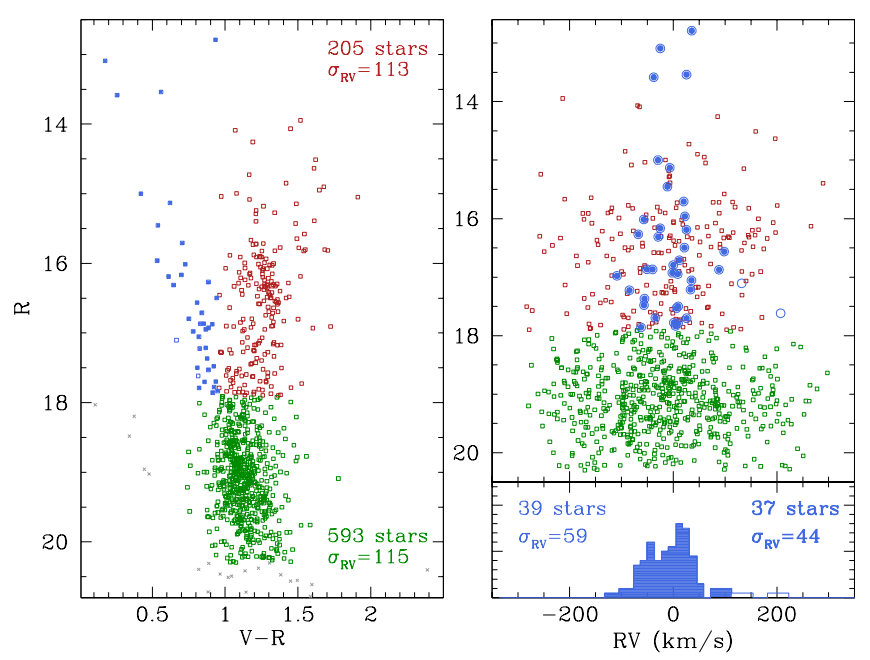

Fig. 6. Same as Fig. 5 for $\mathrm{m} 1 \mathrm{p} 2$ field.

distribution of the global sample. In addition, following the prescription of Ness et al. (2013b), we provide the mean galactocentric radial velocity $\left(V_{\mathrm{GC}}\right)$ by correcting the mean heliocentric value for the Sun motion with respect to the Galactic center.

\section{Discussion and conclusions}

We have measured radial velocities for several hundred bulge stars in each of four fields located within $l= \pm 1.5^{\circ}$ and $b=$ $\pm 2^{\circ}$, with the IFS spectrograph MUSE at the VLT. All the fields are confined within a projected radius of 280 parsecs from the Galactic center, assuming the latter at $8 \mathrm{kpc}$ from the Sun.

The aim of this work is to assess the presence of a large peak in velocity dispersion in this inner region of the Galaxy, previously identified by Zoccali et al. (2014) based on GIBS
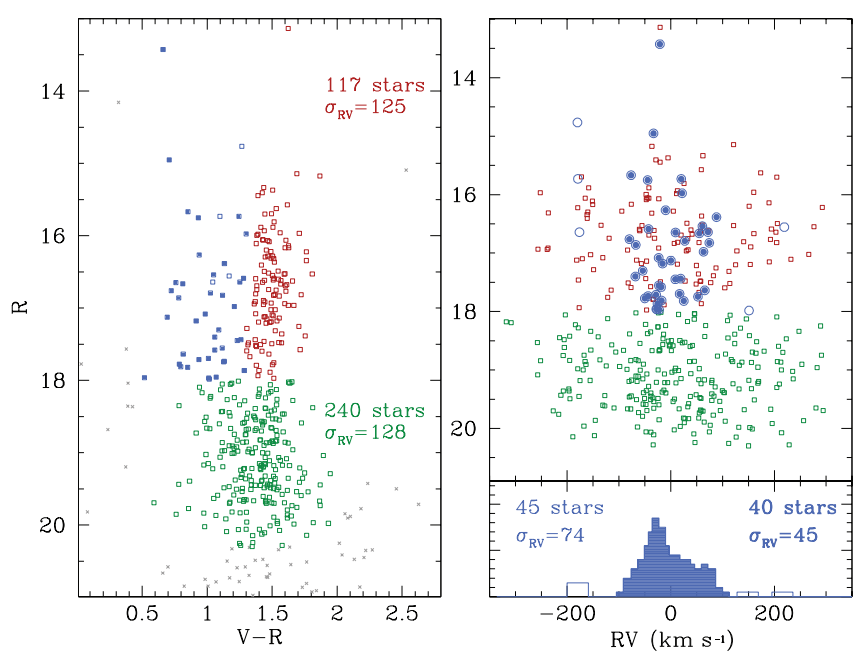

Fig. 7. Same as Fig. 5 for p0p2 field.
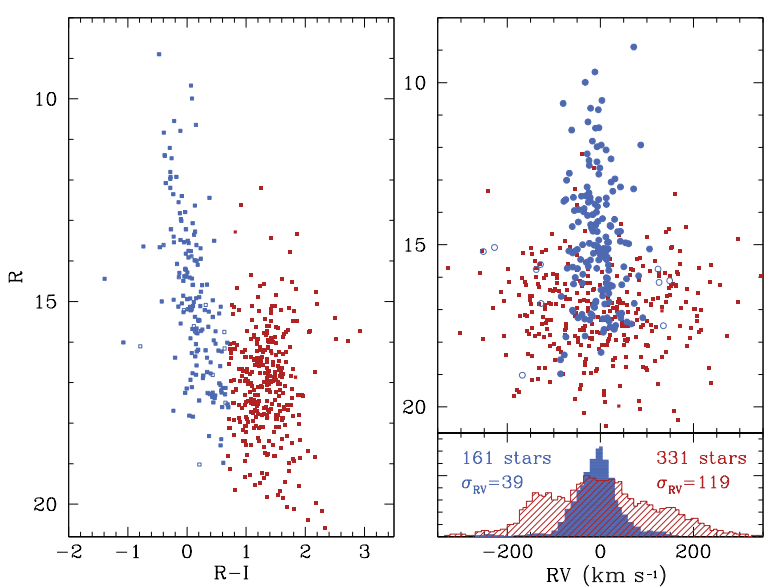

Fig. 8. Same as Fig. 5 for p1m1 field. In this case the bulge MS falls below the limit magnitude, and therefore bulge RGB stars can be separated from disk MS stars by means of a simple color cut.

Table 3. Mean heliocentric and galactocentric radial velocity, and velocity dispersion measured for the bulge stars in the observed fields.

\begin{tabular}{lccccc}
\hline \hline Field & $\begin{array}{c}\left\langle V_{\text {Helio }}\right\rangle \\
\left(\mathrm{km} \mathrm{s}^{-1}\right)\end{array}$ & $\begin{array}{c}\left\langle V_{\mathrm{GC}}\right\rangle \\
\left(\mathrm{km} \mathrm{s}^{-1}\right)\end{array}$ & $\begin{array}{c}\sigma \\
\left(\mathrm{km} \mathrm{s}^{-1}\right)\end{array}$ & $\begin{array}{c}N_{\mathrm{b}} / N_{\text {tot }} N_{\text {tot }} \\
(\%)\end{array}$ & \\
\hline p0m2 & $0 \pm 4.4$ & +9.8 & $135 \pm 3.1$ & 79.5 & 1203 \\
m1p2 & $-14 \pm 4.6$ & -8.8 & $125 \pm 3.3$ & 87.5 & 861 \\
p0p2 & $10 \pm 7.5$ & 19.2 & $137 \pm 5.3$ & 82.4 & 496 \\
p1m1 & $1 \pm 6.6$ & 14.7 & $119 \pm 4.7$ & 67.3 & 502 \\
\hline
\end{tabular}

survey, and to constrain its shape (see Fig. 1 for a zoom of the inner region of the velocity dispersion map derived in that work). Figure 10 (bottom) shows the central velocity dispersion peak as measured in the five innermost GIBS fields (black and gray small points, at $b=-2^{\circ}$ and $b=-1^{\circ}$, respectively) and as predicted in other Galactic positions according to the interpolated surface derived in that paper (black and gray curves). The curve was assumed to be symmetric above and below the Galactic plane, therefore the prediction for negative or positive latitude is identical by definition. The upper panel of Fig. 10 shows the same but for the radial velocity. 


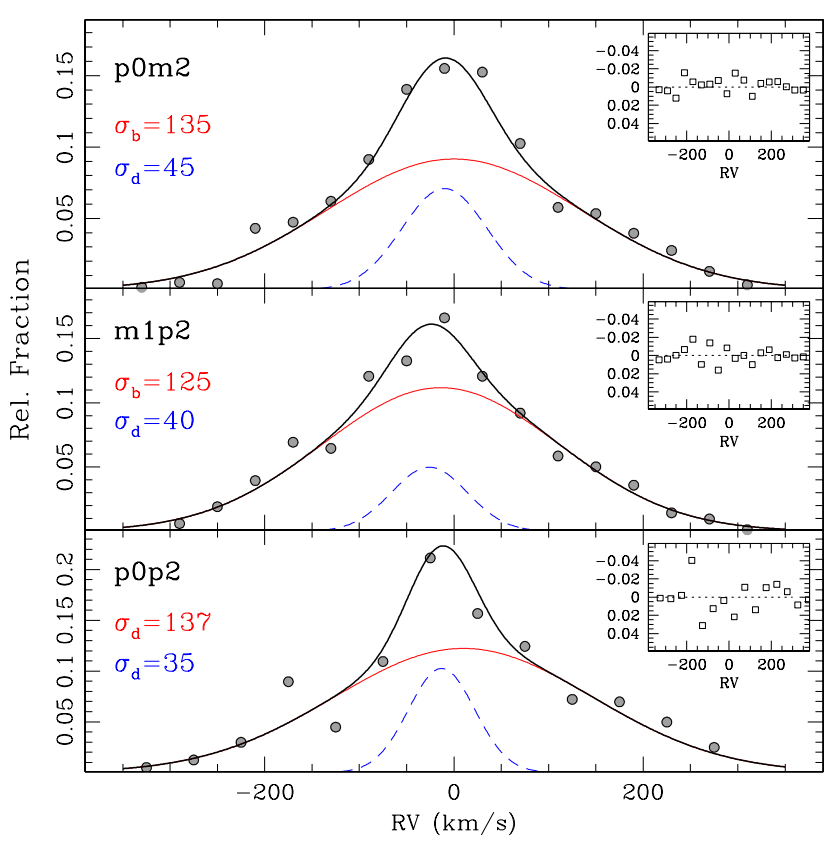

Fig. 9. Normalized heliocentric radial velocity distribution function for all sampled stars observed in the $\mathrm{p} 0 \mathrm{~m} 2$ (top panel), $\mathrm{m} 1 \mathrm{p} 2$ (middle panel), and p0p2 (bottom panel) fields. The best fit to the velocity distribution (solid black line) is obtained by using a combination of two Gaussian (red and blue solid lines) functions whose sigma are also reported in each panel. The residuals of the best fit are shown for each field in the insets.

The new values derived in the present work are plotted in Fig. 10 with large colored symbols. They confirm both the presence of the central velocity dispersion peak, and its absolute value, reaching $\sigma V_{\mathrm{GC}} \sim 140 \mathrm{~km} \mathrm{~s}^{-1}$ at its center. We also confirm that the peak is symmetric above and below the plane, as the two measurements at $(l, b)=\left(0^{\circ},-2^{\circ}\right)$ and $\left(0^{\circ},+2^{\circ}\right)$ are mutually consistent. With the present data we cannot constrain the latitude extension of the peak better than what was done in Zoccali et al. (2014), who found that the peak would disappear at the latitude of Baade's window $\left(b=-4^{\circ}\right)$. We can however constrain the longitude extension of the peak, which we show to be at least as narrow as predicted by GIBS in longitude. In fact, the new fields at $l= \pm 1^{\circ}$ have a velocity dispersion that is lower than the prediction of the GIBS maps.

In Valenti et al. (2016) we have derived maps of stellar projected density and stellar mass from star counts in the PSF catalogs based on the Vista Variable in the Via Láctea survey, using red clump stars as tracers of the total number of stars (and stellar mass). We found the presence of a peak in stellar density in the inner few degrees of the Galaxy, which is reproduced here in Fig. 11. This demonstrates that the sudden increase in velocity dispersion is likely due to the presence of a large concentration of stars (i.e. mass) in the inner Galaxy. The Galactic position and spatial extension of the peak roughly coincides with the sigma peak characterized here, even if its detailed shape is somehow different. In particular, while the sigma peak is still rather sharp at $b= \pm 2^{\circ}$, the peak in star counts is already very shallow at these latitudes. On the other hand, while the conversion from observed velocity and mass requires dynamical modeling, as it requires a hypothesis on the orbit distribution and possible orbits anisotropy, it is qualitatively expected that the effect of a mass concentration on the stellar velocity is felt down to some distance from the mass source. Hence it is not surprising that the

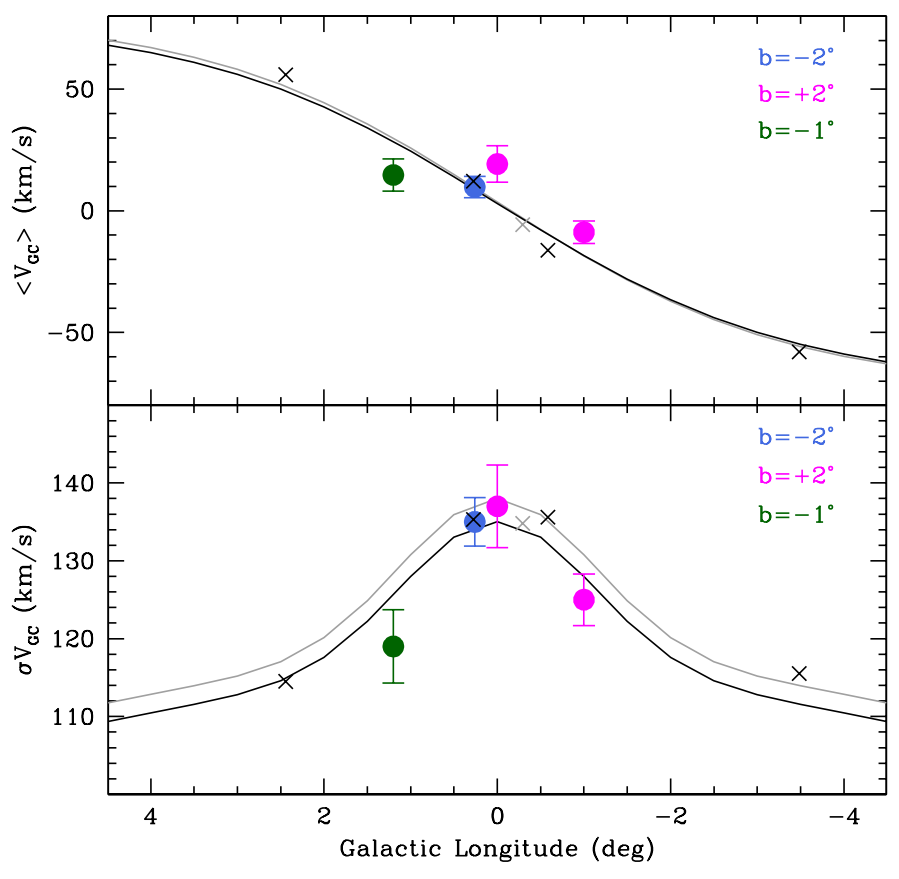

Fig. 10. Mean galactocentric radial velocity (top panel) and velocity dispersion (bottom panel) as a function of the Galactic longitude, for different latitudes as listed in the labels. Big color symbols refer to fields observed with MUSE, whereas small crosses mark the innermost GIBS fields at $b=-2^{\circ}$ (black) and $b=-1^{\circ}$ (gray). The solid lines represent the expected trend of the radial velocity and velocity dispersion according to the maps derived in Zoccali et al. (2014, Eqs. 1 and 2). Error bars are derived from the sampling.

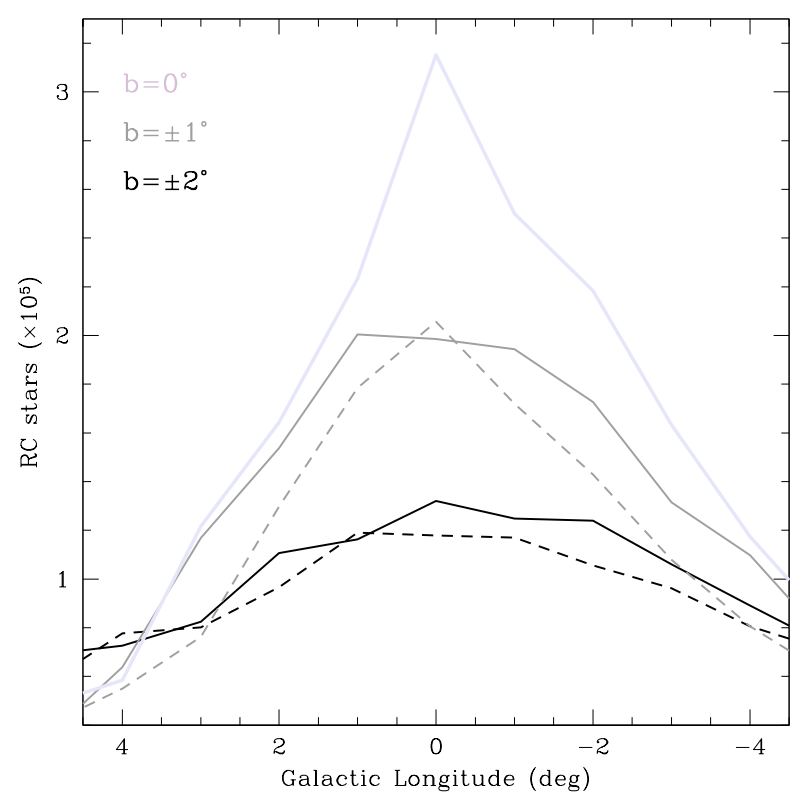

Fig. 11. Profile of the stellar projected density, as traced by RC stars, in the inner few degrees of the Galactic bulge, from Valenti et al. (2016). The figure is a zoom of their Fig. 4, with solid and dashed lines referring to negative and positive latitudes, respectively.

sigma peak is more spatially extended than the stellar density peak.

One thing that deserves further study is the fact that, according to Zoccali et al. (2017), metal-poor stars slightly dominate the stellar density at $b=1^{\circ}$ (their Fig. 7), but the velocity dis- 
persion is higher for metal-rich stars in the same field (their Fig. 12). This is clear evidence that the conversion between velocity dispersion and mass involves at least another parameter (the anisotropy of the orbit distribution) and that this parameter is different for metal-poor and metal-rich stars. The data provided here should be included in the (chemo)-dynamical models of the Galaxy (e.g., Di Matteo et al. 2015; Debattista et al. 2017; Portail et al. 2017; Fragkoudi et al. 2018) in order to properly take into account the mass distribution of the inner few degrees of the Milky Way.

Acknowledgements. EV, MZ, OAG, DM, and MR gratefully acknowledge the Aspen Center for Physics where this work was partially completed. The Aspen Center for Physics is supported by the National Science Foundation grant PHY1066293. During their stay in Aspen, MZ and DM were partially supported by a grant from the Simons Foundation. Support for MZ and DM is provided by the BASAL CATA Center for Astrophysics and Associated Technologies through grant PFB-06, and the Ministry for the Economy, Development, and Tourism's Programa Iniciativa Científica Milenio through grant IC120009, awarded to Millenium Institute of Astrophysics (MAS). EV and MZ also acknowledge support from FONDECYT Regular 1150345. DM acknowledges support from FONDECYT Regular 117012.

\section{References}

Babusiaux, C. 2016, PASA, 33, 026

Babusiaux, C., Gómez, A., Hill, V., et al. 2010, A\&A, 519, A77

Bacon, R., Accardo, M., \& Adjali, L. 2010, in Ground-based and Airborne Instrumentation for Astronomy III, Proc. SPIE, 7735, 773508

Debattista, V. P., Ness, M., Gonzalez, O. A., et al. 2017, MNRAS, 469, 1587
Dékány, I., Minniti, D., Catelan, M., et al. 2013, ApJ, 776, L19

Di Matteo, P., Gómez, A., Haywood, M., et al. 2015, A\&A, 577, A1

Fragkoudi, F., Di Matteo, P., Haywood, M., et al 2018, A\&A, in press DOI: 10.1051/0004-6361/201732509

Freeman, K., Ness, M., Wylie-de-Boer, E., et al. 2013, MNRAS, 428, 3660

Gonzalez, O. A., Rejkuba, M., Zoccali, M., et al. 2012, A\&A, 543, A13

Gran, F., Minniti, D., Saito, R. K., et al. 2016, A\&A, 591, A145

Hill, V., Lecureur, A., Gomez, A., et al. 2011, A\&A, 534, A80

Howard, C. D., Rich, R. M., Clarkson, W., et al. 2009, ApJ, 702, L153

Ness, M., Freeman, K., Athanassoula, E., et al. 2012, ApJ, 756, 22

Ness, M., Freeman, K., Athanassoula, E., et al. 2013a, MNRAS, 430, 836

Ness, M., Freeman, K., Athanassoula, E., et al. 2013b, MNRAS, 432, 2092

Ness, M., Zasowski, G., Johnson, J. A., et al. 2016, ApJ, 819, 2

Pietrukowicz, P., Kozłowski, S., Skowron, J., et al. 2015, ApJ, 811, 113

Portail, M., Wegg, C., Gerhard, O., \& Ness, M. 2017, MNRAS, 470, 1233

Rich, R. M., Reitzel, D. B., Howard, C. D., \& Zhao, H. 2007, ApJ, 658, L29

Robin, A. C., Bienaymé, O., Fernández-Trincado, J. G., \& Reylé, C. 2017, A\&A, 605, A1

Rojas-Arriagada, A., Recio-Blanco, A., Hill, V., et al. 2014, A\&A, 569, A103

Sbordone, L., Bonifacio, P., Castelli, F., \& Kurucz, R. L. 2004, Mem. Soc. Astron. It. Sup., 5, 93

Shen, J., Rich, R. M., Kormendy, J., et al. 2010, ApJ, 720, L72

Stetson, P. B. 1987, PASP, 99, 191

Valenti, E., Zoccali, M., Gonzalez, O. A., et al. 2016, A\&A, 587, L6

Weilbacher, P.M., Streicher, O. \& Urrutia, T. 2012, in Software and Cyberinfrastructure for Astronomy II, Proc. SPIE, 8451, 84510B

Zoccali, M., \& Valenti, E. 2016, PASA, 33, 025

Zoccali, M., Gonzalez, O. A., Vasquez, S., et al. 2014, A\&A, 562, A66

Zoccali, M., Vasquez, S., Gonzalez, O. A., et al. 2017, A\&A, 599, A12 